\title{
The effect of corruption on bidding behavior in first-price auctions
}

\author{
Leandro Arozamena ${ }^{\mathrm{a}, *}$, Federico Weinschelbaum ${ }^{\mathrm{b}}$ \\ a Universidad Torcuato Di Tella and CONICET, Saenz Valiente 1010, C1428BIJ Buenos Aires, Argentina \\ ${ }^{\mathrm{b}}$ Universidad de San Andrés, Vito Dumas 284, B1644BID Victoria, Buenos Aires, Argentina
}

\section{A R T I C L E I N F O}

\section{Article history:}

Received 23 March 2007

Accepted 4 November 2008

Available online 17 November 2008

\section{JEL classification:}

C72

D44

L14

Keywords:

Auctions

Corruption

Right-of-first-refusal

\begin{abstract}
A B S T R A C T
When the owner of an object sells it through an auction run by an agent of hers, corruption may appear. In a first-price auction, corruption can make honest bidders more or less aggressive, or their behavior can remain unchanged. We identify sufficient conditions for each of the three possibilities. We analyze the effects of corruption on efficiency, bidders' welfare and expected revenue. Our results apply as well to the situation-unrelated to corruption-where one of the bidders is granted a right of first refusal.
\end{abstract}

(c) 2008 Elsevier B.V. All rights reserved.

\section{Introduction}

A huge and increasing volume of goods, services and financial instruments are sold and procured through auctions, both in the private and in the public sector. The use of auctions holds a number of advantages that explain its popularity. One of them is the fact that tendering processes are more transparent than their alternatives (e.g. bargaining), as they can be regulated by a fixed and objective set of rules. However, most auctions are not organized and run by the owner of the object on sale. Usually, that task is delegated to an agent. Given this separation between the owner and the auctioneer, corruption may appear. The auctioneer may be tempted to enter into an agreement with one of the bidders to tilt the auction in her favor. Considering the pervasive use of auctions, such corrupt practices can have significant economic consequences.

When a sealed-bid auction is chosen, quite frequently these corrupt deals are reached so that the favored bidder has access to privileged information about her rivals' bids and can adjust her own behavior according to that information. Transparency International (2005), for instance, develops a number of archetypical examples of corruption in construction projects. A key part of what they claim are their most relevant examples is the fact that confidential information about prices quoted by rivals is provided to some potential contractors in exchange for a compensation. Ingraham (2005) examines a scheme used in New York City School Construction Authority auctions whereby one of the bidders was effectively allowed to win by matching the best rival offer, even though bids were supposed to be submitted simultaneously: the favored bidder's offered price was announced last by the official in charge of the auction, and was a "magic number," just enough to win the contract. Lengwiler and Wolfstetter (2007) report further examples of similar corrupt practices that were uncovered in the bidding processes for a new airport in the Berlin area, for the construction of a major power station in Singapore, and a tunnel in Slovenia, among others.

\footnotetext{
* Corresponding author. Tel.: +541151697300; fax: +541151697348.

E-mail addresses: larozamena@utdt.edu (L. Arozamena), fweinsch@udesa.edu.ar (F. Weinschelbaum).
} 
In this paper, we examine a particular form of corruption like the one described in the previous examples. Within a single-object, private-value sealed-bid auction with risk-neutral bidders, we study the possibility that the auctioneer and one bidder reach an agreement whereby, after receiving all bids, (i) the former will reveal to the latter all of her rivals' bids, and (ii) the favored bidder will be allowed to modify her original bid upwards or downwards if she wishes to do so. If this deal exists, then bids are effectively submitted in a sequential, not a simultaneous way.

Our exercise can be interpreted in more than one way. In this paper, we do not model how the corrupt agreement between the auctioneer and one bidder is reached, but concentrate on its influence on the auction itself. We assume that the agreement is arrived at before the auction. We could consider our setup as part of a more complete, two-stage model of corruption, where, first, who the favored bidder will be and how she will compensate the auctioneer are determined, and, second, the auction takes place. Our analysis is useful for any such model. Alternatively, the prior agreement could be part of a special relationship, or one that exceeds the context of the individual auction under study. This is particularly relevant for the case of public procurement, ${ }^{1}$ where officials in charge of the auction may have been captured by parties interested not just in one but in a series of public contracts.

The situation that we model can be interpreted as well as the result of a practice that is unrelated to corruption. One of the bidders may openly and publicly hold the right to match the highest bid that any of her rivals may submit-she may have been awarded that right in a previous contract. That is the arrangement known as right of first refusal, ${ }^{2}$ frequently employed, for example, in share transactions, lease contracts, partnerships and professional sports. It is quite common that a firm favors, by awarding such a clause, a preferred supplier with whom it has a long-standing relationship. Shareholders of close corporations and joint ventures usually hold the right to match any bid for the shares of their fellow shareholders or venturers. In the U.S., the Petroleum Marketing Practices Act establishes that a distributor wishing not to renew a franchise agreement must offer the current franchisee a right of first refusal on any third-party offer. See Walker (1999) for these and many other examples of the use of right-of-first-refusal clauses in a variety of contracts.

We are interested in the effect of the form of corruption described above-or, alternatively, of the existence of a right-offirst-refusal clause ${ }^{3}$ - on how bidders behave and on the auction's welfare and revenue properties. In particular, we want to ascertain how unfavored bidders adjust their behavior in the presence of corruption, i.e. whether they bid more or less aggressively than in a standard, corruption-free auction. This could turn out to be crucial for the effect of this form of corruption on the auction's result.

In a second-price auction, since bidding her own valuation is a weakly dominant strategy for every bidder, this form of corruption has no effect. In a first-price auction, however, the situation is more complex. We show below that an honest bidder can become more or less aggressive or her behavior can remain unchanged in the presence of a corrupt deal. In Proposition 1, we provide sufficient conditions for each of the three possibilities. Furthermore, we establish the extent to which the most commonly used distributions satisfy one of those conditions. As a corollary, we find a result that may be of independent interest: those sufficient conditions also determine the curvature of bidding functions in standardi.e. corruption-free-first-price auctions.

We then move on to assess the effect of this form of corruption on efficiency, revenue and bidders' welfare. If the ability to bid last knowing her rival bids is conferred to a bidder, then the first-price auction will not be efficient. In addition, we show that honest bidders' expected utilities are lower with corruption (Proposition 2) than in a standard first-price auction. The coalition between the auctioneer and the favored bidder always generates a positive surplus to split between the two by reaching a corrupt deal (Proposition 3). As for expected revenue, it cannot be higher with than without corruption in "regular" cases, but we provide an example of a "nonregular" case where corruption raises the expected price received by the seller.

Other papers have dealt with related forms of corruption. In particular, Jones and Menezes (1995), Lengwiler and Wolfstetter (2007), Burguet and Perry (2007) and Menezes and Monteiro (2006) consider cases where a corrupt arrangement, just as in this paper, implies revealing to one of the bidders what her rivals have bid. However, their analyses differ from the one we present in several respects. In Jones and Menezes (1995), bidders are not aware of the possibility of corruption when choosing their bids, so bidding behavior remains unaltered by assumption. ${ }^{4}$ Menezes and Monteiro (2006) consider a situation where the auctioneer approaches the winning bidder offering the chance to lower her bid (while still winning the auction) in exchange for a bribe. Lengwiler and Wolfstetter (2007) study the case where the auctioneer can approach the highest bidder in the way described above or, alternatively, approach the second highest bidder and allow her to raise her bid and become the winner. ${ }^{5}$ In this paper, on the contrary, who the auctioneer may conspire with is independent of the auction's result. That is, their agreement is reached before the auction takes place. The identity of the favored bidder is also determined before the auction in Burguet and Perry (2007). They focus on the

\footnotetext{
${ }^{1}$ Although we model the auction as a selling mechanism, all of our results apply as well to procurement auctions.

2 The right of first refusal has been studied in Walker (1999), Burguet and Perry (2004), Bikhchandani et al. (2005), Arozamena and Weinschelbaum (2006), Lee (2008) and Choi (forthcoming).

${ }^{3}$ For the sake of clarity, we will stick to the corruption interpretation in what follows.

${ }^{4}$ In addition, they consider a setting where bidders draw their valuations from uniform distributions. We prove below that, for such distributions, even if bidders were aware of the possibility of corruption their behavior would remain unaltered.

5 Lengwiler and Wolfstetter (2007) call "type-I" ("type-II") corruption the case where the auctioneer allows the favored bidder to change her original bid downwards (upwards).
} 
two-bidder case, and the favored bidder is allowed to change her original bid upwards to win the auction in cases where she would otherwise lose. ${ }^{6}$ Furthermore, they do not concentrate on the effect corruption has on the auction, but rather on the consequences of how the auctioneer and the favored bidder share the gains from corruption. We extend the analysis to the $N$-bidder case and are able to provide a fuller characterization of the effect of corruption on bidding behavior and auction performance. ${ }^{7}$ Finally, Compte et al. (2005), consider the situation where the auctioneer reveals the winning bid to all participants and allows them to compete for the chance to resubmit their bids. Their focus, then, is on bribing competition and its effects.

While our interest here limits to the case where a single-dimensional object is auctioned, another strand in the literature focuses on the possibility of corruption in multidimensional procurement auctions. Specifically, in addition to the price, the object being procured has a quality dimension that affects the procurer's welfare. The procurer delegates the assessment of quality on an agent, and the scope for corruption is thereby created. Celentani and Ganuza (2002) and Burguet and Che (2004) examine different forms of corruption in that procurement environment.

Our analysis is related as well to the literature on favoritism in auctions. In this literature, some bidders are favored for reasons other than corruption-e.g. in a procurement auction, domestic or local firms may be given preferential treatment when facing foreign rivals. Laffont and Tirole (1991) and Vagstad (1995) study this issue in multidimensional auctions, where favoritism may appear when the auctioneer assesses product quality. McAfee and McMillan (1989), Branco (1994) and Naegelen and Mougeot (1998) examine single-dimensional auctions, where price-preferences may be used. If the identity of the favored bidder is known to all, our results hold and our model may be viewed as a very particular form of favoritism.

The paper is organized as follows. In Section 2, we present the auctioning context and provide sufficient conditions to characterize the effect of corruption on honest bidders' behavior. Then, we ascertain the extent to which standard distributions satisfy each of those sufficient conditions. In Section 3, we study the consequences of corruption on efficiency, welfare and revenue. We conclude in Section 4. Proofs are provided in Appendix C.

\section{The model}

The owner of a single, indivisible object is selling it through an auction ${ }^{8}$ organized and run by an agent of hers. There are $N$ bidders $^{9}$ whose valuations $v_{i}(i=1, \ldots, N)$ for the object are distributed identically and independently according to the c.d.f. $F$ with support on the interval $[v, \bar{v}]$ and a density $f$ that is positive and bounded on the whole support. The context is, then, one of independent private values. We will assume below that $F$ is logconcave. ${ }^{10}$ For future use, let $\alpha\left(v_{i}\right)=F\left(v_{i}\right) / f\left(v_{i}\right)$. Note that the logconcavity of $F$ means that $\alpha$ is increasing. All bidders, the auctioneer and the owner are risk neutral.

We will focus below on sealed-bid auctions with no reserve prices. Given the fact that the owner of the object being sold and the auctioneer are not the same, there is scope for corruption. The auctioneer may tilt the auction in favor of one of the bidders ${ }^{11}$ in exchange for a compensation. The exact form this collusion may take is open to multiple possibilities. Here, we concentrate on one particular case. We assume that the auctioneer has reached an agreement with one bidder before the auction takes place whereby she will reveal to that bidder all of her rivals' bids, ${ }^{12}$ and then allow her to modify her original bid upwards or downwards if she wishes to do so. In essence, a bidder that is awarded such an advantage would take part in a sequential auction, where all of her rivals would bid in the first stage and she would bid in the second.

If such a deal exists, there is one "dishonest" bidder, who will have the advantage of bidding last. Her $(N-1)$ "honest" rivals are aware of the fact that one of the bidders holds this advantage. ${ }^{13}$ Our main objective is to compare equilibrium bidding behavior and auction performance when this agreement takes place with what would happen in the absence of the deal.

The effect of corruption on bidding behavior will clearly differ according to the sealed-bid auction format. In a secondprice auction, for instance, bidding her own valuation is a weakly dominant strategy for every bidder without corruption, and, of course, remains so once corruption appears. Thus, bidding behavior remains unaltered for all bidders, both honest

\footnotetext{
${ }^{6}$ In their setting, whether type-I or type-II corruption is allowed is relevant to how the auctioneer and the favored bidder share their joint surplus. Here, we do not focus on surplus-sharing. Since the favored bidder moves last, her initial bid is meaningless. Thus, allowing one or both types of corruption renders the same results.

7 They consider the case where bidders' valuations are distributed asymmetrically, while we concentrate on a symmetric environment. With two bidders (with or without symmetry) the game that corruption generates is dominance solvable, as will be noted below. With $N$ bidders, dominance solvability is lost.

${ }^{8}$ All of our results, however, are applicable as well to the case of procurement auctions.

9 The number of bidders is taken as given, and participation is assumed to be costless. The case of costly participation and an endogenous number of bidders is analyzed in the working paper version.

${ }^{10}$ Logconcavity of the c.d.f. function holds for most well known distributions, such as the uniform, normal, logistic, extreme value, chi-squared, chi, exponential, Laplace, Pareto and any truncation of these distributions. For details see Bagnoli and Bergstrom (2005).

11 We are not allowing for the possibility of collusion between the bidders (see Hendricks and Porter, 1989, for a general analysis of this phenomenon), but only between one bidder and the auctioneer.

12 It is immaterial whether the auctioneer reveals to the favored bidder all or only the highest of her rivals' bids.

13 With $N>2$, given our symmetric setup, whether honest bidders are aware of the identity of their dishonest rival or not is irrelevant. In the working paper version, we consider the case where honest bidders are not necessarily certain that a corrupt deal exists, but only attach a given probability to it.
} 
and dishonest. Information about rivals' bids is useless in this auction format: the form of corruption we are examining has no effect in this case. ${ }^{14}$

\subsection{The first-price auction}

In a first-price auction corruption has a significant effect. If a bidder colludes with the auctioneer and learns her rivals' bids, she may have an incentive to change her original bid. The comparison between bidding behavior with and without corruption becomes significant.

If no bidder is favored, we have a standard first-price auction. Let $b(v)$ be the bidding function corresponding to a symmetric equilibrium without corruption in our context, and let $\phi(b)$ be its inverse. Each bidder's expected utility maximization problem is then

$$
\max _{b}\left(v_{i}-b\right) F^{(N-1)}(\phi(b))
$$

The first-order condition of this problem, under symmetric bidding behavior, becomes ${ }^{15}$

$$
\phi-b=\frac{F(\phi)}{(N-1) f(\phi) \phi^{\prime}}
$$

The differential equation (1) characterizes the symmetric equilibrium in strictly monotone strategies.

We now turn to the first-price auction in the presence of corruption. ${ }^{16}$ We start by examining the behavior of the last mover. Let $d$ be the dishonest bidder, and let $b^{h}$ be the highest bid submitted by an honest bidder $\left(\right.$ i.e. $\left.b^{h}=\max _{i \neq d} b_{i}\right)$. Let $b_{d}$ be $d$ 's revised bid. If, according to her original bid, $d$ is winning the auction, then she will revise her bid downwards and set $b_{d}=b^{h}+\varepsilon .{ }^{17}$ If she is losing the auction, there are two possibilities. If $v_{d}>b^{h}$, then she will again set $b_{d}=b^{h}+\varepsilon$ and win the auction. If $b^{h}>v_{d}$, then she will submit a revised bid $b_{d}<b^{h}$ (she may leave her original bid unchanged), and lose.

Viewing the auction from the standpoint of an honest bidder that faces a colluding rival, this means that the former will have to bid above the latter's valuation to win. In other words, she will be competing against the dishonest rival's valuation instead of competing against her bid.

Let $b_{i}^{c}:[v, \bar{v}] \longrightarrow \mathbb{R}$ be bidder $i$ 's bidding function when $i$ is honest and there is a favored rival. We will look for an equilibrium in strictly increasing strategies that is symmetric among honest bidders. That is, we will look for an equilibrium where $b_{i}^{c}(v)=b^{c}(v)$ for all $i$ such that $i$ is honest. For convenience, we will use the inverse bidding function, $\phi^{c}(\cdot)$. An honest bidder $i$ with valuation $v_{i}$ who faces a dishonest rival and all of whose honest rivals bid according to the inverse bidding function $\phi^{c}(\cdot)$ that is strictly increasing will choose her bid by solving the following expected utility maximization problem:

$$
\max _{b}\left(v_{i}-b\right) F^{(N-2)}\left(\phi^{c}(b)\right) F(b)
$$

By bidding $b$, an honest bidder will defeat any particular honest rival with probability $F\left(\phi^{c}(b)\right)$, and she will defeat the dishonest bidder with probability $F(b)$. Then, $F^{(N-2)}\left(\phi^{c}(b)\right) F(b)$ is the probability of winning the auction by bidding $b$ for an honest bidder. The first-order condition resulting from (2) is

$$
v_{i}-b=\frac{F\left(\phi^{c}\right) F(b)}{(N-2) F(b) f\left(\phi^{c}\right) \phi^{c \prime}+F\left(\phi^{c}\right) f(b)}
$$

In a symmetric equilibrium we will have $v_{i}=\phi^{c}(b)$, and the first-order condition becomes

$$
\phi^{c}-b=\frac{F\left(\phi^{c}\right) F(b)}{(N-2) F(b) f\left(\phi^{c}\right) \phi^{c \prime}+F\left(\phi^{c}\right) f(b)}
$$

Appendix A provides a proof that the differential equation (3) has a strictly increasing solution. Then, (3) characterizes the inverse bidding function $\phi^{c}(b)$ in a symmetric equilibrium. ${ }^{18}$

As usual in the first-price auction, $\phi^{c}(\underline{v})=\underline{v}$. Our main objective is to establish how the function $\phi^{c}(b)$ characterized by (3) compares with $\phi(b)$, which results from (1). That is, we want to find out whether it is possible to say that with corruption honest bidders become uniformly more aggressive $\left(\phi^{c}(b)<\phi(b)\right.$ for all $b>\underline{v}$ in their common support),

\footnotetext{
14 These conclusions could change if we allowed for corrupt coalitions where the auctioneer and more than one bidder could be involved. Note, besides, that the second-price auction could be more vulnerable to the possibility of collusion among bidders than the first-price auction. See, for example, Robinson (1985) and Marshall and Marx (2007).

${ }^{15}$ To economize on notation, we will omit the argument of the inverse bidding function $\phi$ where possible. It is well known that, in this case, the standard, first-price auction has a unique symmetric equilibrium in strictly monotone strategies. See, for example, Krishna (2002).

${ }^{16}$ Our results also hold in the case where there is a secret reserve price that is distributed according to the same c.d.f. as bidders' valuations. See Elyakime et al. (1994) and Li and Tan (2000) for the study of secret reserve prices in private-value, first-price auctions.

${ }^{17}$ We may assume that $b_{d}=b^{h}$ and, in the event of a tie, the auctioneer chooses the winner. Therefore, she will always choose the bidder she is trying to favor.

${ }^{18}$ The fact that the second-order sufficient condition is satisfied is checked in Appendix B. In general, there is no closed-form solution to ( 3 ). We cannot guarantee that the solution to (3) is unique. However, all the results that follow hold for any solution to that differential equation.
} 
uniformly less aggressive $\left(\phi(b)<\phi^{c}(b)\right.$ for all $b>\underline{v}$ in their common support) or keep their behavior unaltered $\left(\phi(b)=\phi^{c}(b)\right.$ for all $b$ ). In the remainder of this section, we provide sufficient conditions for each of these three possibilities.

As we mentioned above, given the logconcavity of the c.d.f. $F$, we know that $\alpha(v)$ is increasing. We will show in what follows that a key fact to ensure that corruption generates more, equal or less aggressiveness is whether $\alpha(v)$ is strictly convex, linear or strictly concave. Proposition 1 asserts that those are sufficient conditions to determine the effect of corruption on the behavior of an honest bidder.

Proposition 1. If $\alpha(v)$ is strictly convex (linear, strictly concave) then $\phi(b)>\phi^{c}(b)$ (respectively, $\left.\phi(b)=\phi^{c}(b), \phi(b)<\phi^{c}(b)\right)$ for all $b>\underline{v}$ in their common supports.

It follows that $\alpha(v)$ being strictly convex (concave) determines that corruption generates more (less) aggressiveness, while $\alpha(v)$ being linear makes corruption irrelevant for the behavior of an honest bidder. Furthermore, it can be easily verified that $\alpha(v)$ being linear is also a necessary condition for bidding functions to remain unaltered by corruption.

It turns out, in addition, that the curvature of $\alpha(v)$ determines as well the curvature of bidding functions in standard, symmetric first-price auctions in the absence of corruption. This result, which—being unrelated to corruption-may be of independent interest, is established in the following corollary.

Corollary 1. If $\alpha(v)$ is strictly convex (linear, strictly concave) then $b(v)$, the bidding function in a standard, symmetric first-price auction, is strictly concave (respectively, linear, strictly convex).

Let us use Corollary 1 to gain a better understanding of the results in Proposition 1 . To simplify, assume $N=2$ and $\underline{v}=0$, and compare $\phi(b)$ with $\phi^{c}(b)$. From (1) and (3), $\phi(b)-b=\alpha(\phi(b)) / \phi^{\prime}(b)$ and $\phi^{c}(b)-b=\alpha(b)$. Corruption shifts the rival's valuation that the honest bidder faces marginally (the valuation she ties with by bidding $b$ ) from $\phi(b)$ to $b$, which is lower. Whether the honest bidder will then have incentives to be more or less aggressive depends on the relationship between $\alpha(\phi(b)) / \phi^{\prime}(b)$ and $\alpha(b)$. From the logconcavity of $F(v)$, we know that $\alpha(b) \leqslant \alpha(\phi(b))$. That is, if the dishonest bidder's inverse bidding function had a slope at $b$ which equaled $\phi^{\prime}(b)$, then the honest bidder would necessarily become more aggressive with corruption. However, there is a counteracting effect: with corruption, the dishonest bidder bids her true valuation, so her inverse bidding function has a slope equal to 1 . Since $\phi^{\prime}(b)>1$, this change in the marginal behavior of her rival provides the honest bidder with incentives to become less aggressive.

Suppose $\alpha(v)$ is linear. Corollary 1 tells us, then, that $\phi$ is linear, so $\alpha(\phi) / \phi^{\prime}=\alpha\left(\phi^{\prime} b\right) / \phi^{\prime}$. From the linearity of $\alpha(v)$, the last expression equals $\alpha(b)$. That is, the ratio $\alpha(\phi) / \alpha(b)$ is constant and equal to the (constant) slope of the inverse bidding function, $\phi^{\prime}$. The two effects mentioned above exactly offset each other in this case. Proposition 1, thus, tells us that the first effect outweighs the second in the case where $\alpha(v)$ is strictly convex, and the opposite result obtains when $\alpha(v)$ is strictly concave. It also tells us that this generalizes to any number of bidders.

Remark 1. Proposition 1 is useful to establish a result that is different but related to our main question. Let $\widetilde{\phi}(b)$ be the inverse bidding function of an honest bidder who is certain that all of her rivals will learn her bid and then be allowed to rebid. ${ }^{19}$ Thus, such a bidder would have to bid higher than the highest valuation among her rivals' to win the auction. It is straightforward to show that the analog of (3) in this case is

$$
\widetilde{\phi}(b)-b=\frac{F(b)}{(N-1) f(b)}=\frac{\alpha(b)}{N-1}
$$

Corollary 2. If $\alpha(v)$ is strictly convex (linear, strictly concave) then $\widetilde{\phi}(b) \leqslant \phi^{c}(b)<\phi(b)$ (respectively, $\widetilde{\phi}(b)=\phi^{c}(b)=\phi(b)$, $\left.\widetilde{\phi}(b) \geqslant \phi^{c}(b)>\phi(b)\right)$ for all $b>\underline{v}$ in their common supports, where all inequalities are strict if $N>2 .{ }^{20}$

The result follows immediately by comparing (3) and (4) and using Proposition 1 and Lemma 2 in Appendix C.

We have provided sufficient conditions to characterize how bidding behavior is affected by a corrupt arrangement between the auctioneer and one of the bidders. As noted, how an honest bidder's behavior will be influenced by the existence of corruption will be determined by the concavity or convexity of $\alpha(v)$. The next natural question is, of course, if most of the commonly used distribution functions imply that $\alpha(v)$ is concave or convex. The next subsection briefly deals with this issue.

\subsection{The effect of corruption for commonly used distributions}

It is easy to verify that $\alpha(v)$ being linear is equivalent to $F(v)$ being a power function distribution, i.e. $F(v)=$ $(v-\underline{v})^{k} /(\bar{v}-\underline{v})^{k}$ for some $k>0 .^{21}$ We can fully characterize, then, the family of distributions where our necessary and

\footnotetext{
19 This case is equivalent to ours only if $N=2$.

20 Porter and Shoham (2005) independently proved the part of this result that refers to the comparison between $\phi(b)$ and $\widetilde{\phi}(b)$.

${ }^{21}$ For these distributions, $\alpha(v)=(v-\bar{v}) / k$. Note that, except in the case where $k=1$ (i.e. when $F(v)$ is the uniform distribution), the Proof of Proposition 1 does not apply to distributions in this family, since $f(\underline{v})$ is not positive and bounded. However, the validity of the proposition can be verified by computing the bidding functions directly.
} 
sufficient condition for corruption not to have any effect on honest bidding behavior applies. Bidding functions are given by $b^{p}(v)=[1 /(k(N-1)+1)][\underline{v}+k(N-1) v]$.

Note that

$$
\alpha^{\prime}(v)=1-\frac{F(v)}{f(v)} \frac{f^{\prime}(v)}{f(v)}
$$

If $F$ is strictly logconcave (logconvex), then $F(v) / f(v)$ will be strictly increasing (decreasing). By the same token, if $f$ is strictly logconcave (logconvex), then $f^{\prime}(v) / f(v)$ will be strictly decreasing (increasing). Recall that we have assumed that $F$ is logconcave. We can combine these possibilities to provide simple sufficient conditions for the concavity or convexity of $\alpha(v)$.

Remark 2. (a) If $f(v)$ is logconcave and decreasing $\left(f^{\prime}(v)<0\right)$, then $\alpha(v)$ is convex.

(b) If $f(v)$ is logconvex and increasing $\left(f^{\prime}(v)>0\right)$, then $\alpha(v)$ is concave.

The exponential distribution is an example of part (a) in Remark 2, while $F(v)=\left[\mathrm{e}^{-\lambda} /\left(1-\mathrm{e}^{-\lambda}\right)\right]\left(\mathrm{e}^{\lambda v}-1\right)$, with support $[0,1]$, is an example of (b). In addition, it can be verified as well if $\alpha(v)$ is concave or convex for any of the standard distributions. Straightforward calculations show that in the cases of the logistic, Laplace and Pareto distributions, for instance, $\alpha(v)$ is strictly convex. Hence, for all of them the existence of corruption makes an honest bidder more aggressive. As for the normal distribution, however, $\alpha(v)$ is not strictly concave or convex on all of its support. Still, it is only strictly concave more than six standard deviations below its mean, and it is strictly convex in the remainder of the support. Hence, most truncations of the normal distribution would generate a strictly convex $\alpha(v)$ and more aggressiveness with corruption.

\section{Efficiency and welfare}

We have studied above how a specific form of corruption affects the behavior of honest bidders in first-price auctions and provided sufficient conditions to assess how they adjust their bids when facing a rival that is dishonest. In this section we analyze the effect of this form of corruption on efficiency, the seller's revenue, and bidders' welfare.

It will be useful, for expositional purposes, to split the total effect that the presence of corruption has on the different welfare measures into two effects, the "direct effect" and the "perception effect." The direct effect is the one that the presence of corruption has on the expected utility of any party to the auction holding the bidding functions of all honest bidders constant. It reflects, then, the fact that there exists a dishonest bidder, but does not include any variation in honest bidders' strategies. The perception effect, on the other hand, is the change in the expected utility of any party that is exclusively due to the fact that honest bidders change their behavior when there is a dishonest bidder. Notice that when $\alpha(v)$ is linear, the perception effect vanishes, since there is no change in how honest bidders behave.

Let us start with efficiency. In the absence of corruption, and keeping all our other assumptions, it is well known that the first-price auction is efficient. A natural consequence of our previous analysis, however, is that the first-price auction will be inefficient with corruption. Every honest bidder will shade her bid (i.e. her equilibrium bid will be lower than her actual valuation). Let $v_{d}$ be the dishonest bidder's valuation, as above, and $v^{h}=\max _{i \neq d} v_{i}$. Given that honest bidders have increasing symmetric equilibrium strategies $b^{c}(v)$, the highest honest bid will be $b^{h}=b^{c}\left(v^{h}\right)$. As Fig. 1 shows, if the dishonest bidder is allowed to examine bids and resubmit her own by the auctioneer and $v_{d} \in\left[b^{c}\left(v^{h}\right), v^{h}\right)$, she will win the auction in spite of the fact that her valuation is not the highest.

This means as well that the first-price auction is worse in efficiency terms than the second-price auction in the presence of corruption.

Let us turn now to bidders' welfare. Regarding the expected utility of honest bidders, we know that the direct effect is always negative: given the behavior of honest rivals, the presence of corruption means that an honest bidder will lose in some cases where she would have won. This is a consequence of the fact that a dishonest bidder is allowed by the auctioneer to revise her original offer upwards to match the highest original bid. ${ }^{22}$ As for the perception effect, it is not positive in the cases where honest bidders do not become less aggressive in the presence of corruption (that is, if $\alpha(v)$ is linear or strictly convex). Thus, in these cases, combining both effects, it is certain that corruption is detrimental to the expected utility of an honest bidder in the auction. With corruption, any honest bidder wins with a lower probability and, when she wins, she has to pay a (weakly) higher price.

When $\alpha(v)$ is strictly concave, the perception effect is positive, since all honest rivals bid strictly less aggressively. We therefore have two effects of opposite signs. However, Proposition 2 establishes that the expected utility of honest bidders falls with the presence of corruption in all cases, even in those where $\alpha(v)$ is not always concave or convex.

Proposition 2. The expected utility of an honest bidder is lower when there is corruption.

As regards the coalition of the dishonest bidder and the auctioneer, how they will distribute any gains they may reap from their agreement is undetermined in our analysis. So we will refer to their joint surplus as the "expected utility of the

\footnotetext{
${ }^{22}$ Note that with $N=2$ there are no further effects on the honest bidder's welfare.
} 


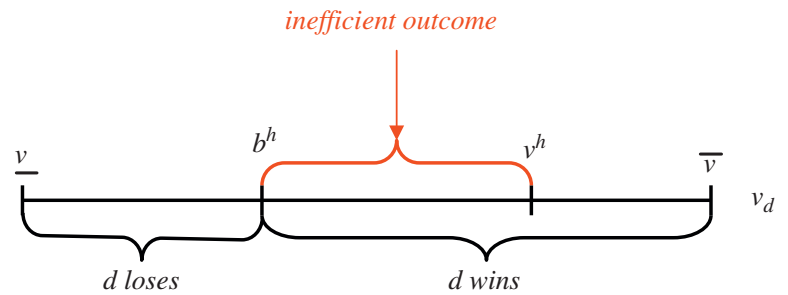

Fig. 1.

coalition." Suppose first that the coalition exists, i.e. there is corruption. We may then ask how the surplus of the coalition varies because of the honest bidders' reaction to the existence of corruption-in other words, we may look purely at the perception effect. The answer follows straightforwardly from Proposition 1: the perception effect is positive (negative) for the coalition when $\alpha(v)$ is strictly concave (strictly convex), since honest bidders respond by bidding less aggressively (more aggressively) when there is corruption; needless to say, the coalition's surplus is invariant when $\alpha(v)$ is linear.

A related question is whether there will be a positive surplus for the coalition, i.e. if its expected utility is larger when they reach an agreement (and honest bidders react to its existence) than if they reach no agreement and the auction is corruption-free. Here, the direct and perception effects are again combined. Clearly, the coalition is better off whenever honest bidders do not become more aggressive with corruption (that is, if $\alpha(v)$ is linear or strictly concave). The dishonest bidder wins with a higher probability for any valuation (the direct effect is positive) and never pays a higher price (the perception effect is also positive). For the case of $\alpha(v)$ strictly convex, the perception effect is negative. Is it possible that honest bidders become so much more aggressive with corruption that there is no gain for the coalition in reaching an agreement? The following proposition provides a negative answer.

Proposition 3. The coalition's expected utility (i.e. the joint surplus of the auctioneer and the dishonest bidder) is larger with than without corruption. ${ }^{23}$

Note that Proposition 3, just as Proposition 2, applies in all cases, even in those where $\alpha(v)$ is not always linear, strictly concave or strictly convex on $[\underline{v}, \bar{v}]$.

Finally, let us examine the consequences of corruption on expected revenue. The direct effect is always negative. The dishonest bidder may choose to revise her bid upwards or downwards. When the former occurs, only the identity of the winner changes, but not the price paid. When the latter occurs, however, the price paid to the owner falls. The perception effect is zero when $\alpha(v)$ is linear, and negative if $\alpha(v)$ is strictly concave. So we certainly know that the presence of corruption will lower expected revenue in those two cases.

When $\alpha(v)$ is strictly convex, though, honest bidders become more aggressive, so the two effects go in opposite directions. In particular, when the change in honest bidders' behavior is large enough, it may be the case that the seller's expected revenue grows with the presence of corruption. We do know, however, that expected revenue with corruption has to be lower than expected revenue without corruption in the cases that are usually called "regular," that is, in those cases where a bidder's "virtual" valuation $v-[(1-F(v)) / f(v)]$ is increasing in her "true" valuation $v$. In regular cases, the standard first-price auction is revenue-maximizing within the class of mechanism where the object is always sold. Within this class, to maximize revenue the object should be awarded to the bidder with the highest virtual valuation. For any regular case, that is just the bidder with the highest valuation, and that is the bidder who wins in a standard first-price auction. When there is corruption, with positive probability the winner does not have the highest valuation, so expected revenue has to be lower.

This result, though, does not hold for nonregular cases. Moreover, honest bidders can become so much more aggressive in the presence of corruption that expected revenue may grow, as we show in the following example.

Example 1. Suppose $N=2$ and valuations are distributed according to the Pareto distribution with parameters 1 and 0.75 (i.e. $\left.F(v)=1-(1 / v)^{0.75}\right)$, with support on $[1,+\infty)$. Without corruption, the seller's revenue is 3 , whereas it is 3.7431 with corruption.

In the working paper version we extend this analysis to the case where participation in the auction is costly and the number of bidders is endogenous. There we show that, given that corruption is detrimental to the expected welfare of honest bidders, it reduces participation in the auction. Therefore, as compared to the case where the number of bidders is exogenous, corruption makes the first-price auction less efficient and further reduces expected revenue, while it creates an even larger expected surplus for the coalition of the auctioneer and the dishonest bidder.

\footnotetext{
${ }^{23}$ Another interesting question may be whether reaching an agreement with the auctioneer is better for a bidder than a situation where another bidder becomes the favored one. The combination of Propositions 2 and 3 provides a positive answer.
} 


\section{Conclusion}

We have analyzed above how a specific form of corruption affects the behavior of honest bidders in sealed-bid auctions. Assuming that the auctioneer has reached an agreement with one of the bidders by which the latter will be shown all of her rival's bids and will be allowed to resubmit her bid accordingly, we have provided sufficient conditions to assess how honest bidders adjust their bids in a first-price auction. Those conditions determine whether honest bidders will react by behaving more, equally or less aggressively. In any case, the first-price auction becomes inefficient with corruption. We have established as well that corruption hurts honest bidders, and that the coalition of the auctioneer and the favored bidder always has a positive expected surplus to divide between its two members if they reach an agreement. Corruption is detrimental to expected revenue in "regular" cases, though not necessarily so in "nonregular" ones.

Let us emphasize again that, even though we have been very precise in terms of the advantages that corruption confers to a dishonest bidder in our analysis, we have not studied the negotiation between such a bidder and the auctioneer that leads to a corrupt arrangement. Our results, then, can be regarded as relevant to any specific model for such negotiation in the context of sealed-bid auctions. They are relevant as well, as explained above, to the situation where one of the bidders is granted a right of first refusal.

Our analysis could be extended in several directions. First, it would be instructive to allow for the possibility that bidders' valuations be distributed asymmetrically, or to explore the case where bidders are risk averse. Second, the bargaining game between the auctioneer and the favored bidder could be included in the analysis. Finally, we have studied a case where corruption takes a very specific form. The effects of many other forms of corruption on behavior in auctions remain unexplored.

\section{Acknowledgments}

We are grateful to Daniel Aromi, Oscar Barraza, Sushil Bikhchandani, Roberto Burguet, Marco Celentani, Walter Cont, Juan José Ganuza, Yvan Lengwiler, David Levine, Pino Lopomo, Alejandro Manelli, Elmar Wolfstetter, José Wynne, participants at various seminars, the editor, Esther Gal-Or, an associate editor, two anonymous referees and especially to Paul Klemperer for their comments on a previous version of this paper, and to Maximiliano Appendino for excellent research assistance. We acknowledge financial support from FONCyT, PICT 2255.

\section{Appendix A}

This appendix proves that there exists a strictly monotone solution to the differential equation provided by the firstorder condition (3). ${ }^{24}$

Note that if $N=2$ the game is dominance solvable. The first-order condition is then

$$
v_{i}=b+\frac{F(b)}{f(b)}
$$

which characterizes a unique, strictly increasing inverse bidding function as long as $F$ is logconcave.

For $N>2$, the game is not dominance solvable. The proof begins by following the same steps as in Li and Tan (2000). For convenience, we restate the differential equation:

$$
\phi^{c \prime}=\frac{F\left(\phi^{c}\right)\left[1-\frac{f(b)}{F(b)}\left(\phi^{c}-b\right)\right]}{(N-2) f\left(\phi^{c}\right)\left(\phi^{c}-b\right)}
$$

We look for a strictly monotone solution $\phi^{c}(b)$ such that $b, \phi^{c}(b) \in[\underline{v}, \bar{v}]$ and $\phi^{c}(b) \geqslant b$ for all $b$, with the initial condition $\phi^{c}(\underline{v})=\underline{v}$. Hence, our domain is the set $\Delta=\left\{(b, v) \in[\underline{v}, \underline{v}]^{2}: b \leqslant v\right\}$. Both the numerator and the denominator of $(5)$ can be zero in that set, so we cannot use standard existence theorems in this context. The strategy of the proof is then to reduce the domain to a set of pairs $(b, v)$ where standard existence theorems apply and then extend the solution.

We start reducing the domain by excluding all $(b, v)$ such that $b=\underline{v}$. Notice that, within the remainder of the set $\Delta$, the denominator of (5) is zero when $v=b$ and is positive when $v>b$. Let $n(b, v)=[1-(f(b) / F(b))(v-b)]$. For any $v>\underline{v}$, $n(b, v)>0$ if $v=b$. In addition, $n(b, v)$ is strictly decreasing in $v$. Let

$$
\bar{\phi}(b)= \begin{cases}\widehat{v} \text { if } n(b, \widehat{v})=0 & \text { for } \widehat{v} \in(b, \bar{v}) \\ \bar{v} & \text { otherwise }\end{cases}
$$

Then, $b \leqslant \bar{\phi}(b)$, and the inequality is strict if $b \in(\underline{v}, \bar{v})$. We define the smaller domain

$$
\Delta^{*}=\left\{(b, v) \in(\underline{v}, \bar{v}]^{2}: b<v \leqslant \bar{\phi}(b)\right\}
$$

\footnotetext{
${ }^{24}$ We are grateful to an anonymous referee who detected an error in a previous version of this proof and suggested a way to correct it.
} 
On $\Delta^{*}$, we can apply the standard existence and uniqueness theorems (see, for instance, Chicone, 1999, Section 1.10). Then, for any $\left(b_{0}, v_{0}\right) \in \Delta^{*}$, there exists a unique solution $\phi_{0}^{c}(b)$ in a neighborhood of $b_{0}$ such that $v_{0}=\phi_{0}^{c}\left(b_{0}\right)$, and that solution is continuous in $b_{0}$. Furthermore, that solution can be extended to the left and right without losing uniqueness along the $b$ axis, and then be defined over a larger interval. Let $\left(\beta_{0}, \beta_{1}\right)$ be the maximal open interval of existence of the solution. By the extensibility theorem (see Chicone, 1999 , Section 1.10 ), when $b \longrightarrow \beta_{1}$, $\left(b, \phi_{0}^{c}(b)\right.$ ) approaches a point on the boundary of $\Delta^{*}$.

We want to show that $\lim _{b \rightarrow \beta_{1}^{-}} \phi_{0}^{c}(b)=\bar{v}$. If that is not true, then either $\lim _{b \rightarrow \beta_{1}^{-}} \phi_{0}^{c}(b)=\beta_{1}$ or $\lim _{b \rightarrow \beta_{1}^{-}} \phi_{0}^{c}(b)=$ $\bar{\phi}\left(\beta_{1}\right)<\bar{v}$. Assume $\lim _{b \rightarrow \beta_{1}^{-}} \phi_{0}^{c}(b)=\beta_{1}$. As $b$ approaches $\beta_{1}$, the denominator of (5) can be made arbitrarily small, and $\phi_{0}^{c \prime}(b)$ can be made arbitrarily large. This means that, close enough to $\beta_{1}$, it has to be the case that $\phi_{0}^{c}(b)<b$, a contradiction.

Suppose now that $\lim _{b \rightarrow \beta_{1}^{-}} \phi_{0}^{c}(b)=\bar{\phi}\left(\beta_{1}\right)<\bar{v}$. This means that $n\left(\beta_{1}, \lim _{b \rightarrow \beta_{1}^{-}} \phi_{0}^{c}(b)\right)=0$, which implies in turn that $\phi_{0}^{c \prime}(b)$ can be made arbitrarily small by making $b$ approach $\beta_{1}$. Note, however, that by the implicit function theorem

$$
\left.\frac{\mathrm{d} v}{\mathrm{~d} b}\right|_{n(b, v)=0}=-\frac{-\frac{\mathrm{d}}{\mathrm{d} b}\left(\frac{f(b)}{F(b)}\right)(v-b)+\frac{f(b)}{F(b)}}{-\frac{f(b)}{F(b)}}
$$

which is positive given that $F$ is logconcave. Then, $\bar{\phi}^{\prime}\left(\beta_{1}\right)>0$. A function with arbitrarily small slope cannot reach from below a function with a strictly positive slope, so a contradiction obtains again. We conclude then that as $b$ approaches $\beta_{1}$, $\phi_{0}^{c}(b)$ approaches $\bar{v}$.

We then have a unique, strictly monotone extended solution that takes values in the interval $\left[v_{0}, \bar{v}\right.$. We need to extend that function again so that it takes values on the whole interval of valuations, $[\underline{v}, \bar{v}$. To do so, we can take any strictly increasing, differentiable function $\lambda_{0}(b)$ such that $\lambda_{0}(\underline{v})=\underline{v}$ and $\lambda_{0}\left(b_{0}\right)=b_{0}$ (for example, we could choose a linear function). The extended function is, then,

$$
\widetilde{\phi}_{0}^{c}(b)= \begin{cases}\phi_{0}^{c}(b) & \text { if } b \geqslant b_{0} \\ \lambda_{0}(b) & \text { if } b<b_{0}\end{cases}
$$

Let us now take a sequence $\left(b_{0}^{n}, v_{0}^{n}\right)_{n=1}^{\infty}$ such that $b_{0}^{n} \longrightarrow \underline{v}^{+}$and $v_{0}^{n} \longrightarrow \underline{v}^{+}$. For each $n$, we can construct a function $\widetilde{\phi}_{0}^{c n}(b)$ as described above. We thereby obtain a sequence of strictly increasing functions. By Helly's selection theorem, ${ }^{25}$ that sequence has a subsequence that converges pointwise. Take one such subsequence and let $\phi^{c}(b)$ be its limit.

We must show now that $\phi^{c}(b)$ is a solution to (5). We know that

$$
\phi^{c}(b)=\lim _{n \rightarrow \infty}\left[\int_{\underline{v}}^{b_{0}} \lambda_{0}^{\prime}(s) \mathrm{d} s+\int_{b_{0}}^{b} \frac{F\left(\widetilde{\phi}_{0}^{c n}\right)\left[1-\frac{f(s)}{F(s)}\left(\widetilde{\phi}_{0}^{c n}-s\right)\right]}{(N-2) f\left(\widetilde{\phi}_{0}^{c n}\right)\left(\widetilde{\phi}_{0}^{c n}-s\right)} \mathrm{d} s\right]+\underline{v}
$$

Then, by Lebesgue's dominated convergence theorem, ${ }^{26}$

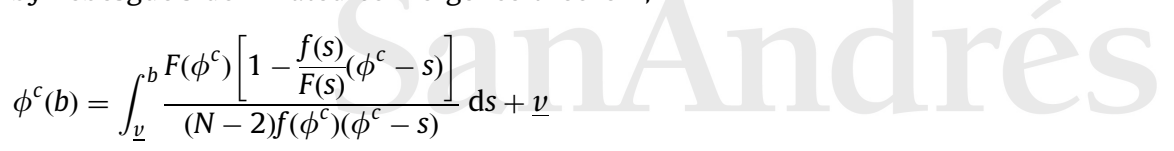

which is (5) in integral form.

\section{Appendix B}

In this appendix we check that the second-order conditions of the maximization problem in (2) are satisfied. ${ }^{27}$ If $N=2$, the first-order condition of that problem can be expressed as

$$
-1+\left(v_{i}-b\right) \frac{f(b)}{F(b)}=0
$$

Since $F$ is logconcave, the derivative of the left-hand side with respect to $b$ is negative, and the second-order condition is satisfied.

If $N>2$, we have to check that the second-order condition is satisfied at the symmetric solution characterized by the first-order condition (3). Given that a bidder's rivals behave according to a strictly increasing bidding function $b^{c}(v)$, with inverse $\phi^{c}(b)$, let $U(v, b)=(v-b) F^{N-2}\left(\phi^{c}(b)\right) F(b)$. Then,

$$
U_{b}(v, b)=-F^{N-2}\left(\phi^{c}(b)\right) F(b)+(v-b)\left[(N-2) F^{N-3}\left(\phi^{c}(b)\right) f\left(\phi^{c}(b)\right) \phi^{c \prime}(b) F(b)+F^{N-2}\left(\phi^{c}(b)\right) f(b)\right]
$$

\footnotetext{
25 See Rudin (1976, p. 167).

${ }^{26}$ See Rudin (1976, p. 321). Notice that the integrand in (6) is bounded if $s>\underline{v}$. Using L'Hôpital's rule, it can be checked that it is also bounded when $s \rightarrow \underline{v}$. Then, Lebesgue's theorem applies.

${ }^{27}$ We acknowledge once again the suggestions of an anonymous referee that improved the exposition in this appendix.
} 
From (3),

$$
(N-2) f\left(\phi^{c}(b)\right) \phi^{c \prime}(b) F(b)+F\left(\phi^{c}(b)\right) f(b)=\frac{F\left(\phi^{c}(b)\right) F(b)}{\phi^{c}(b)-b}
$$

Substituting in the previous expression,

$$
\begin{aligned}
U_{b}(v, b) & =-F^{N-2}\left(\phi^{c}(b)\right) F(b)+(v-b) F^{N-3}\left(\phi^{c}(b)\right) \frac{F\left(\phi^{c}(b)\right) F(b)}{\phi^{c}(b)-b} \\
& =F^{N-2}\left(\phi^{c}(b)\right) F(b)\left[-1+\frac{v-b}{\phi^{c}(b)-b}\right]=F^{N-2}\left(\phi^{c}(b)\right) F(b) \frac{v-\phi^{c}(b)}{\phi^{c}(b)-b}
\end{aligned}
$$

Thus, $U_{b}(v, b)$ is negative (positive) for $b$ such that $v-\phi^{c}(b)<(>) 0$, so it is optimal to choose $b$ such that $v-\phi^{c}(b)=0$.

\section{Appendix C}

In what follows, we provide proofs for the propositions and corollaries in Sections 2 and 3. We start by stating and proving two auxiliary lemmas that will be used in the Proof of Proposition 1.

Lemma 1. For any $b, \alpha(b) \leqslant \alpha\left(\phi^{c}\right) / \phi^{c \prime}$ if and only if

$$
\frac{\alpha(b)}{N-1} \leqslant \phi^{c}-b \leqslant \frac{\alpha\left(\phi^{c}\right)}{\phi^{c \prime}(N-1)}
$$

Naturally, an analogous condition applies reverting the inequalities.

Proof. Notice that, from (3),

$$
\phi^{c}-b-\frac{\alpha(b)}{N-1}=\frac{F\left(\phi^{c}\right) F(b)}{(N-2) F(b) f\left(\phi^{c}\right) \phi^{c \prime}+F\left(\phi^{c}\right) f(b)}-\frac{F(b)}{f(b)(N-1)}
$$

which is nonnegative if and only if

$$
F\left(\phi^{c}\right) f(b)(N-1) \geqslant(N-2) F(b) f\left(\phi^{c}\right) \phi^{c \prime}+F\left(\phi^{c}\right) f(b)
$$

or

$$
F\left(\phi^{c}\right) f(b)-f\left(\phi^{c}\right) \phi^{c \prime} F(b) \geqslant 0
$$

This is true if and only if $\alpha(b) \leqslant \alpha\left(\phi^{c}\right) / \phi^{c \prime}$.

In addition,

$$
\frac{\alpha\left(\phi^{c}\right)}{\phi^{c \prime}(N-1)}-\left(\phi^{c}-b\right)=\frac{F\left(\phi^{c}\right)}{f\left(\phi^{c}\right) \phi^{c \prime}(N-1)}-\frac{F\left(\phi^{c}\right) F(b)}{(N-2) F(b) f\left(\phi^{c}\right) \phi^{c \prime}+F\left(\phi^{c}\right) f(b)}
$$

is nonnegative if and only if

$$
F\left(\phi^{c}\right)\left[(N-2) F(b) f\left(\phi^{c}\right) \phi^{c \prime}+F\left(\phi^{c}\right) f(b)\right] \geqslant f\left(\phi^{c}\right) \phi^{c \prime}(N-1) F\left(\phi^{c}\right) F(b)
$$

or

$$
F\left(\phi^{c}\right)\left[F\left(\phi^{c}\right) f(b)-F(b) f\left(\phi^{c}\right) \phi^{c \prime}\right] \geqslant 0
$$

Again, this is true if and only if $\alpha(b) \leqslant \alpha\left(\phi^{c}\right) / \phi^{c \prime}$.

Lemma 2. If $\alpha(v)$ is strictly convex (linear, strictly concave) then $\alpha(b)<\alpha\left(\phi^{c}\right) / \phi^{c \prime}\left(\right.$ respectively, $\alpha(b)=\alpha\left(\phi^{c}\right) / \phi^{c \prime}, \alpha(b)>$ $\left.\alpha\left(\phi^{c}\right) / \phi^{c \prime}\right)$ for all $b>\underline{v}$.

Proof. We only present the proof for the case where $\alpha(v)$ is strictly convex, since the remaining two cases are analogous. We proceed in two steps:

1. We show first that if $\alpha(\widehat{b})=\alpha\left(\phi^{c}(\widehat{b})\right) / \phi^{c \prime}(\widehat{b})$ for some $\widehat{b}>\underline{v}$, then $\alpha(b)<(>) \alpha\left(\phi^{c}(b)\right) / \phi^{c \prime}(b)$ for $b$ close to but larger (smaller) than $\widehat{b}$. This means that if these two functions cross at $\widehat{b}, \alpha(b) \operatorname{crosses} \alpha\left(\phi^{c}(b)\right) / \phi^{c \prime}(b)$ from above and $\alpha(b)<$ $\alpha\left(\phi^{c}(b)\right) / \phi^{c^{\prime}}(b)$ for all $b>\widehat{b}$. From Lemma 1, we know that $\alpha(\widehat{b})=\alpha\left(\phi^{c}(\widehat{b})\right) / \phi^{c^{\prime}}(\widehat{b})$ if and only if $\alpha(\widehat{b}) /(N-1)=\phi^{c}(\widehat{b})-\widehat{b}$ Then, combining these two equalities yields

$$
\phi^{c \prime}(\widehat{b})-1=\frac{\alpha\left(\phi^{c}(\widehat{b})\right)}{\alpha(\widehat{b})}-1=\frac{\alpha\left(\phi^{c}(\widehat{b})\right)-\alpha(\widehat{b})}{\left[\phi^{c}(\widehat{b})-\widehat{b}\right](N-1)}
$$


The strict convexity of $\alpha(v)$ implies

$$
\phi^{\prime \prime}(\widehat{b})-1>\frac{\alpha^{\prime}(\widehat{b})}{N-1}
$$

Then, $\phi^{c}(b)-b$ is growing faster than $\alpha(b) /(N-1)$ at $b=\widehat{b}$. Resorting to Lemma 1 once more, our claim follows.

2. It is immediate that $\alpha(\underline{v})=\alpha\left(\phi^{c}(\underline{v})\right) / \phi^{c \prime}(\underline{v})=0 .{ }^{28}$ Our second step proves that, for $b$ close to $\underline{v}$, it has to be true that $\alpha(b)<\alpha\left(\phi^{c}\right) / \phi^{c \prime}$. Given our first step, this claim would establish the lemma. Assume, towards a contradiction, that $\alpha(b) \geqslant \alpha\left(\phi^{c}\right) / \phi^{c \prime}$ for all $b \in(\underline{v}, \underline{v}+\delta)$ for some $\delta>0$ arbitrarily small. Take any $b_{0}$ in that interval. Without loss of generality, we can concentrate on the case where $\alpha\left(b_{0}\right)>\alpha\left(\phi^{c}\left(b_{0}\right)\right) / \phi^{c \prime}\left(b_{0}\right)$,since if $\alpha\left(b_{0}\right)=\alpha\left(\phi^{c}\left(b_{0}\right)\right) / \phi^{c \prime}\left(b_{0}\right)$, step 1 implies that $\alpha(b)>\alpha\left(\phi^{c}(b)\right) / \phi^{c \prime}(b)$ for $b$ smaller than but close to $b_{0}$. From Lemma $1, \alpha\left(b_{0}\right) /(N-1)>\phi^{c}\left(b_{0}\right)-b_{0}$. Then, as $\alpha(\underline{v}) /(N-1)=\phi^{c}(\underline{v})-\underline{v}=0$, there has to exist a bid $b_{1} \leqslant b_{0}$ such that $\alpha\left(b_{1}\right) /(N-1)>\phi^{c}\left(b_{1}\right)-b_{1}$ and $\alpha^{\prime}\left(b_{1}\right) /(N-1)>$ $\phi^{c \prime}\left(b_{1}\right)-1$. Since $\alpha\left(b_{1}\right)>\alpha\left(\phi^{c}\left(b_{1}\right)\right) / \phi^{c \prime}\left(b_{1}\right)$, Lemma 1 implies as well that $\phi^{c \prime}\left(b_{1}\right)>\alpha\left(\phi^{c}\left(b_{1}\right)\right) /\left[\left[\phi^{c}\left(b_{1}\right)-b_{1}\right](N-1)\right]$. Therefore,

$$
\frac{\alpha^{\prime}\left(b_{1}\right)}{N-1}>\frac{\alpha\left(\phi^{c}\left(b_{1}\right)\right)-\left[\phi^{c}\left(b_{1}\right)-b_{1}\right](N-1)}{\left[\phi^{c}\left(b_{1}\right)-b_{1}\right](N-1)}
$$

Furthermore, from the strict convexity of $\alpha(v)$,

$$
\frac{\alpha\left(\phi^{c}\left(b_{1}\right)\right)-\alpha\left(b_{1}\right)}{\left[\phi^{c}\left(b_{1}\right)-b_{1}\right](N-1)}>\frac{\alpha^{\prime}\left(b_{1}\right)}{N-1}
$$

For these last two inequalities to hold it has to be the case that $\alpha\left(b_{1}\right)<\left[\phi^{c}\left(b_{1}\right)-b_{1}\right](N-1)$. We thereby conclude that such a $b_{1}$ cannot exist.

Proof of Proposition 1. Again, we only look at the case where $\alpha(b)$ is strictly convex. Lemma 2 implies that

$$
\alpha(b)<\frac{\alpha\left(\phi^{c}(b)\right)}{\phi^{c \prime}(b)}
$$

for any $b>\underline{v}$. The proof proceeds in two steps that are analogous to the ones followed in the proof of Lemma 2.

First, note that if $\phi(\widehat{b})=\phi^{c}(\widehat{b})$ for some $\widehat{b}>\underline{v}$, then (1), (3) and (7) imply that $\phi^{\prime}(\widehat{b})>\phi^{c \prime}(\widehat{b})$. This means that if the two bidding functions cross at some $\widehat{b}>\underline{v}$, then $\phi(b)$ crosses $\phi^{c}(b)$ from below and $\phi(b)>\phi^{c}(b)$ for all $b>\widehat{b}$. Second, we want to show that $\phi(b)>\phi^{c}(b)$ for all $b$ larger than but close to $\underline{v}$. Suppose, towards a contradiction, that there exists some bid $b_{0}>\underline{v}$ such that $\phi(b) \leqslant \phi^{c}(b)$ for all $b \in\left(\underline{v}, b_{0}\right)$. Then, (7) holds again, which implies

$$
\frac{\mathrm{d}}{\mathrm{d} b}\left[\frac{F\left(\phi^{c}(b)\right)}{F(\phi(b))}\right]<0
$$

for all $b \in\left(\underline{v}, b_{0}\right)$. It follows that

$$
\int_{\underline{v}}^{b_{0}} \frac{\mathrm{d}}{\mathrm{d} b}\left[\frac{F\left(\phi^{c}(b)\right)}{F(\phi(b))}\right] \mathrm{d} b<0
$$

or

$$
\frac{F\left(\phi^{c}\left(b_{0}\right)\right)}{F\left(\phi\left(b_{0}\right)\right)}-\lim _{b \rightarrow \underline{v}} \frac{F\left(\phi^{c}(b)\right)}{F(\phi(b))}<0
$$

The second term on the right-hand side of this expression, using L'Hôpital's rule, becomes

$$
\frac{f(\underline{v}) \phi^{c \prime}(\underline{v})}{f(\underline{v}) \phi^{\prime}(\underline{v})}=1
$$

since $\phi^{\prime}(\underline{v})=\phi^{c \prime}(\underline{v})$, as can be easily verified. Therefore, it must be true that

$$
\frac{F\left(\phi^{c}\left(b_{0}\right)\right)}{F\left(\phi\left(b_{0}\right)\right)}<1
$$

and then $\phi\left(b_{0}\right)>\phi^{c}\left(b_{0}\right)$, a contradiction.

Proof of Corollary 1. From (1), we know that

$$
\phi^{\prime}=\frac{\alpha(\phi)}{(N-1)(\phi-b)}
$$

\footnotetext{
${ }^{28}$ Note that, $\phi^{c \prime}(\underline{v})$ cannot be smaller than 1 , since otherwise we would have $\phi^{c}(b)<b$ for $b$ close to $\underline{v}$.
} 
Differentiating this expression,

$$
\phi^{\prime \prime}=\frac{\alpha^{\prime}(\phi) \phi^{\prime}(\phi-b)-\alpha(\phi)\left(\phi^{\prime}-1\right)}{(N-1)(\phi-b)^{2}}
$$

Substituting according to (8), the numerator of this expression becomes

$$
\frac{\alpha^{\prime}(\phi) \alpha(\phi)}{N-1}-\alpha(\phi)\left(\phi^{\prime}-1\right)=\frac{\alpha(\phi)}{N-1}\left[\alpha^{\prime}(\phi)-\left(\phi^{\prime}-1\right)(N-1)\right]
$$

From (8), we know that

$$
(N-1)\left(\phi^{\prime}-1\right)=\frac{\alpha(\phi)-(N-1)(\phi-b)}{\phi-b}
$$

Proposition 1 implies that if $\alpha(v)$ is linear, then $(N-1)(\phi-b)=\alpha(b)$. Then,

$$
(N-1)\left(\phi^{\prime}-1\right)=\frac{\alpha(\phi)-\alpha(b)}{\phi-b}=\alpha^{\prime}(\phi)
$$

where the last equality follows from the linearity of $\alpha(v)$. It follows that the expression in (9) equals zero, and we conclude that $\phi^{\prime \prime}=0$, and so does $b^{\prime \prime}$. Analogously, if $\alpha(v)$ is strictly convex (strictly concave), then, from Proposition 1 , $(N-1)(\phi-b)>(<) \alpha(b)$, so

$$
(N-1)\left(\phi^{\prime}-1\right)<(>) \frac{\alpha(\phi)-\alpha(b)}{\phi-b}<(>) \alpha^{\prime}(\phi)
$$

where the last inequality follows from the strict convexity (strict concavity) of $\alpha(v)$. Therefore, the expression in (9) is positive (negative), so $\phi^{\prime \prime}>(<) 0$. Then, $b^{\prime \prime}<(>) 0$.

Proof of Proposition 2. For the proof, we use standard mechanism design tools. Let us construct the direct revelation mechanism that is equivalent to the first-price auction with corruption. Let $x^{c}(v)$ be the probability with which an honest bidder that announces a valuation $v$ wins, and let $t^{c}(v)$ be the expected price she has to pay. Then, the expected utility of an honest bidder with valuation $v$ is $U^{c}(v)=v x^{c}(v)-t^{c}(v)$. By the envelope theorem, $U^{c \prime}(v)=x^{c}(v)$, so $U^{c}(v)=U^{c}(\underline{v})+$ $\int_{v}^{v} x^{c}(s) \mathrm{d} s$. Since $U^{c}(\underline{v})=0$, it follows that

$$
U^{c}(v)=\int_{\underline{v}}^{v} x^{c}(r) \mathrm{d} r=\int_{\underline{v}}^{v} F^{(N-2)}(r) F\left(b^{c}(r)\right) \mathrm{d} r
$$

Without corruption, we know that the expected utility is

$$
U(v)=\int_{\underline{v}}^{v} F^{(N-1)}(r) \mathrm{d} r
$$

Given that $b^{c}(v)<v$, we have $U^{c}(v)<U(v)$ for any $v>\underline{v}$.

Proof of Proposition 3. Let $\widehat{U}^{c}(v)$ be the expected utility of the coalition when the dishonest bidder's valuation is $v$ and there is corruption. Then,

$$
\widehat{U}^{c}(v)=\int_{\underline{v}}^{\phi^{c}(v)}\left(v-b^{c}(r)\right) F^{(N-2)}(r) f(r) \mathrm{d} r
$$

Now

$$
\int_{\underline{v}}^{\phi^{c}(v)}\left(v-b^{c}(r)\right) F^{(N-2)}(r) f(r) \mathrm{d} r>\int_{\underline{v}}^{v}(v-r) F^{(N-2)}(r) f(r) \mathrm{d} r
$$

where the last expression is the expected utility of a dishonest bidder when all honest rivals bid their own valuations, and the inequality follows from the fact that $b^{c}(r)<r$ for all $r$ and $\phi^{c}(v)>v$. But the right-hand side of this inequality is just the expected utility of the coalition in a second-price auction, which is unaffected by corruption. Without corruption, the auctioneer gets zero, and the expected utility of the coalition is just the expected utility of any bidder with valuation $v$. In a first-price auction without corruption that expected utility is exactly the same as in a second-price auction, since both auction formats are payoff-equivalent in that case. Then, we conclude that for any valuation $v>\underline{v}$ of the dishonest bidder there is a gain from corruption for the coalition of the auctioneer and the dishonest bidder.

\section{References}

Arozamena, L., Weinschelbaum, F., 2006. A note on the suboptimality of right-of-first-refusal clauses. Economics Bulletin 4 (24), 1-5. Bagnoli, M., Bergstrom, T., 2005. Log-concave probability and its applications. Economic Theory 26, 445-469.

Bikhchandani, S., Lippman, S., Ryan, R., 2005. On the right-of-first-refusal. Advances in Theoretical Economics 1 (1) (Article 4 ).

Branco, F., 1994. Favoring domestic firms in procurement contracts. Journal of International Economics 37, 65-80.

Burguet, R., Che, Y., 2004. Competitive procurement with corruption. RAND Journal of Economics 35, 50-68. 
Burguet, R., Perry, M., 2004. Preferred suppliers and vertical integration in auction markets. Mimeo, Institut d'Analisi Economica and Rutgers University. Burguet, R., Perry, M., 2007. Bribery and favoritism by auctioneers in sealed-bid auctions. The B.E. Journal of Theoretical Economics 7 (1) (Contributions, Article 23).

Celentani, M., Ganuza, J., 2002. Competition and corruption in procurement. European Economic Review 43, $1273-1303$.

Chicone, C., 1999. Ordinary Differential Equations with Applications. Springer, Berlin.

Choi, A., forthcoming. A Rent extraction theory of right of first refusal. Journal of Industrial Economics.

Compte, O., Lambert-Mogiliansky, A., Verdier, T., 2005. Corruption and competition in procurement. RAND Journal of Economics 36 , 1-15.

Elyakime, B., Laffont, J., Loisel, P., Vuong, Q., 1994. First-price sealed-bid auctions with secret reservation prices. Annales d'Economie et de Statistique 34, 115-141.

Hendricks, K., Porter, R., 1989. Collusion in auctions. Annales d'Economie et de Statistique 15/16, 1415-1444.

Ingraham, A., 2005. A test for collusion between a bidder and an auctioneer in sealed-bid auctions. Contributions to Economic Analysis and Policy 4 (1) (Article 10).

Jones, C., Menezes, F., 1995. Auctions and corruption: how to compensate the auctioneer. Mimeo, Australian National University.

Krishna, V., 2002. Auction Theory. Academic Press, New York.

Laffont, J., Tirole, J., 1991. Auction design and favoritism. International Journal of Industrial Organization 9, 9-42.

Lee, J., 2008. Favoritism in asymmetric procurement auctions. International Journal of Industrial Organization 26, $1407-1424$.

Lengwiler, Y., Wolfstetter, E., 2007. Auctions and corruption: an analysis of bid rigging by a corrupt auctioneer. Mimeo, Universität Basel and HumboldtUniversität.

Li, H., Tan, G., 2000. Hidden reserve prices with risk-averse bidders. Mimeo, University of Southern California.

Marshall, R., Marx, L., 2007. Bidder collusion. Journal of Economic Theory 133, 374-402.

McAfee, R.P., McMillan, J., 1989. Government procurement and international trade. Journal of International Economics 26, $291-308$.

Menezes, F., Monteiro, P., 2006. Corruption and auctions. Journal of Mathematical Economics 42, 97-108.

Naegelen, F., Mougeot, M., 1998. Discriminatory public procurement policy and cost reduction incentives. Journal of Public Economics 67, $349-367$.

Porter, R.W., Shoham, Y., 2005. On cheating in sealed-bid auctions. Decision Support Systems 39, 41-54.

Robinson, M., 1985. Collusion and the choice of auction. RAND Journal of Economics 16, 141-145.

Rudin, W., 1976. Principles of Mathematical Analysis, third ed. McGraw-Hill, New York.

Transparency International, 2005. Preventing corruption in construction projects: examples of corruption. Mimeo.

Vagstad, S., 1995. Promoting fair competition in public procurement. Journal of Public Economics 58, 283-307.

Walker, D., 1999. Rethinking rights of first refusal. Stanford Journal of Law, Business and Finance 5, 1-58. 